\title{
Incidence, Causes, and Correlates of Maternal Near-Miss Morbidity: a multi-centre cross-sectional study
}

\author{
Samuel A. Oppong ${ }^{*}, 1$, Ashura Bakari ${ }^{2}$, April J. Bell ${ }^{3}$, Yemah Bockarie ${ }^{4}$, Joseph A Adu ${ }^{5}$, \\ Cornelius A. Turpin ${ }^{6}$, Samuel A Obed ${ }^{1}$, Richard M Adanu ${ }^{7}$, and Cheryl A. Moyer ${ }^{3}$ \\ 1) School of Medicine and Dentistry, University of Ghana, Accra, Ghana \\ 2) Suntresu Government Hospital, Ghana Health Service, Kumasi, Ghana \\ 3) University of Michigan, Ann Arbor, Michigan, USA \\ 4) Cape Coast Teaching hospital, Cape Coast, Ghana \\ 5) School of Medical Sciences, University of Cape Coast, Cape Coast, Ghana \\ 6) School of Medical Sciences, Kwame Nkrumah University of Science and Technology, Kumasi, \\ Ghana \\ 7) School of Public Health, University of Ghana, Accra, Ghana
}

\section{Abstract}

Objective: To explore the incidence and factors associated with maternal near-miss.

Design: Cross-sectional study with an embedded case-control study

Setting: Three tertiary referral hospitals in southern Ghana

Population: All women admitted to study facilities with pregnancy-related complications or for birth.

Methods: An adapted version of the WHO Maternal Near Miss Screening Tool was used to identify maternal near-miss cases. These were compared with unmatched controls (uncomplicated deliveries) in a ratio of 1:2.

Main outcome measures: Incidence of maternal near-miss, maternal near-miss-maternal mortality ratio, and cause of and factors associated with maternal near-miss.

\footnotetext{
*Corresponding Author Samuel Antwi Oppong, Department of Obstetrics \& Gynaecology, School of Medicine and Dentistry, University of Ghana, PO Box 4236, Accra, Ghana, wak72@yahoo.com.

Contribution to authorship

$\mathrm{SAO}, \mathrm{AB}, \mathrm{AJB}, \mathrm{RMA}$ and $\mathrm{CAM}$ conceived the idea and developed the research question, $\mathrm{SAO}, \mathrm{AB}, \mathrm{AJB}, \mathrm{YB}, \mathrm{JAA}, \mathrm{CAT}$ and $\mathrm{SO}$ conducted the experiment to obtain the data; $\mathrm{SAO}, \mathrm{AB}, \mathrm{AJB}$ and CAM analysed the data, $\mathrm{SAO}$ and CAM wrote the first draft of the manuscript, and all authors reviewed and approved the final manuscript before submission.

Disclosures

The authors disclose that they have no conflict of interest. Completed disclosure of interest forms are available to view online as supporting information.

Ethical approval

This study and its components were reviewed and approved by the IRBs of University of Ghana for KBTH site (MS-Et/M.7 P4.5/214-2015 on March 10, 2015), University of Cape Coast for CCTH (UCCIRB/EXT/2015/02 on April 8, 2015) and at the KATH (CHRPE/ AP/021/15 on 25th January 2015) as well as the University of Michigan (HUM00097103 on February 16,2015) which was the data coordinating center.
} 
Results: Out of 8,433 live births, 288 maternal near-miss cases and 62 maternal deaths were identified. 454 healthy controls were recruited for comparison. Maternal near-miss and maternal death incidence ratios were 34.2 (95\% CI:30.2 - 38.1) and 7.4 (95\% CI:5.5 - 9.2) per 1000 live births respectively with a maternal near-miss-mortality ratio of 4.6:1. Cause of near-miss was preeclampsia/eclampsia (41.0\%), haemorrhage (12.2\%), maternal sepsis (11.1\%), and ruptured uterus $(4.2 \%)$. Major factor associated with maternal near-miss was maternal fever within the seven days before birth (OR:5.95 95\%CI:3.754 - 9.424). Spontaneous onset of labor was protective against near miss OR:0.09 95\% CI: $0.057-0.141$ )

Conclusion: For every maternal death, there were nearly five maternal near-misses. Women having a fever in the seven days prior to delivery were six times more likely to experience a nearmiss than women not having fever.

\section{Tweetable abstract:}

Maternal near-miss exceed maternal death by 5:1, with the leading cause of maternal near-miss was preeclampsia/eclampsia.

\section{Keywords}

maternal near-miss; maternal mortality; maternal near-miss indicators

\section{Introduction}

Low income countries, especially those in sub-Saharan Africa (SSA), continue to bear a disproportionate burden of maternal morbidity and mortality[1]. Ghana is one of the countries in sub-Saharan Africa that failed to reach the millennium development goal for maternal health, which was a target of no more than 185 maternal deaths per 100,000 live births. Ghana ended 2015 with a maternal mortality ratio of nearly twice the target: 350 per 100,000 live births[2]. Pregnant women in Ghana continue to die from largely preventable causes, including haemorrhage, hypertensive diseases, and abortion-related complications [3-7]. These mortality figures are only a small part of the story. For every woman who dies, there are many others who survive severe, life-threatening complications that may have long-lasting sequelae [8-10].

Morbidity and mortality can be seen as a continuum. Maternal health can be described as ranging from normal pregnancy with no complications at one end through mild non-lifethreatening complications, life-threatening complications, and death at the other end of the continuum. As such, a maternal near-miss has been defined as "a woman who nearly died from a life-threatening complication of pregnancy, delivery, or up to 42 days after termination of pregnancy but survived" [11]. Depending on the context and the specific criteria used, the incidence of maternal near-miss ranges from $0.6 \%$ to more than $30 \%$ of all live births[12]. There has been one previous study from Ghana which estimated that maternal near-misses outnumbered mortality at a ratio of about 3 to 1 in a single setting[7]. Thus, the true incidence of maternal near-miss in Ghana is not known, and the cause of as well as factors associated with maternal near-miss are also not well understood. This is in 
stark contrast to the well-developed literature describing the causes of maternal mortality[37].

As mortality events become less common, maternal near-misses are likely to become an increasingly important metric by which quality of care can be measured. We explored maternal near-misses at three of Ghana's four tertiary care obstetric units to identify the burden, cause of, as well as factors associated with maternal near-miss morbidity.

The primary aim of this study was to estimate the incidence of maternal near-miss morbidity per 1000 live births. The secondary aims were; 1) to determine the maternal near-miss : mortality ratio and, 2) to use an embedded case-control study to determine factors associated with maternal near-miss.

\section{Method}

Study design

We performed a multi-center, cross-sectional study with an embedded case-control study.

\section{Setting}

This study was conducted at three tertiary referral hospitals in southern Ghana over a fourmonth period (April 1 to July 31, 2015). The study sites were the maternal and neonatal units of the Korle-bu Teaching hospital (KBTH) in Accra, Cape Coast Teaching hospital (CCTH) and Komfo Anokye Teaching hospital (KATH) in Kumasi. KBTH is located in Accra, the capital city of Ghana, with a population of about 3.2 million [13]. It is the largest referral hospital in Ghana, overseeing approximately 11,000 deliveries annually and serving as the teaching hospital for the School of Medicine and Dentistry, University of Ghana. KATH is located in Kumasi, the second largest city in Ghana, with a population of about 2 million[13]. KATH serves as the referral center for most of mid-Ghana, overseeing approximately 11,000 deliveries per year, and serving as the teaching hospital affiliated with the Kwame Nkrumah University of Science and Technology School of Medical Sciences (KNUST-SMS). CCTH is located to the west of Accra at the coastal town of Cape Coast, about 100 miles from Accra. It serves as the main referral hospital for most of the rural Central and parts of the Western region of Ghana, overseeing approximately 2,800 deliveries per year and serving as the teaching hospital of the University of Cape Coast, School of Medical Sciences (UCC-SMS).

\section{Determining the number of live births}

Summary statistics were collected reflecting the total number of deliveries, live births, and maternal deaths for the study period from each of the three participating hospitals' records. In addition, trained research assistants reviewed all admission and delivery records as well as caesarean operations record books to record the total number of deliveries during the study period. 


\section{Identifying and classifying maternal near-misses}

Each day, research assistants reviewed the admission and delivery records for all pregnant women aged 18 to 49 who delivered at one of the three study sites or were referred with pregnancy or delivery-related complications up to 42 days post termination of the pregnancy. Complications were defined as any indication in the medical record that a woman had experienced a pregnancy- or delivery-related complication (haemorrhage; preeclampsia, eclampsia, or other hypertensive disorder; premature rupture of membranes; premature delivery; oligohydramnios; gestational diabetes; miscarriage; complication from abortion; obstructed labor / failure to progress; malposition; shoulder dystocia; emergency cesarean section; placenta previa; or any other noted pregnancy or delivery complication). This list of complications was used across all three study sites.

All records were screened for eligibility using the modified version of the WHO Maternal Near Miss Screening Tool[14] which was simplified for use in settings without all of the laboratory tests and intervention procedures available as included in the full WHO instrument (supplemental file S1). Participants were assessed for the presence of specific symptom-based criteria (such as severe haemorrhage, severe preeclampsia, eclampsia, sepsis or systemic infection or ruptured uterus, assessed by attending obstetrician/gynaecologist), intervention-based criteria (such as use of blood products, laparotomy, or admission to the intensive care unit) or organ dysfunction-based criteria (such as cardiovascular, respiratory, renal, haematologic, hepatic, neurologic, or uterine dysfunction). A positive response to any of the above qualified a woman as a 'near-miss'. This classification was double checked by an attending physician (consultant) at each study location. Women were excluded if they died during childbirth or were unwilling to provide consent. Incidental maternal deaths would have been excluded, however no incidental maternal deaths were identified during the study period.

\section{Identification of 'controls' for embedded case-control study}

As described above, research assistants went through each medical record and completed a unique screening form for each patient, indicating which of the near-miss criteria were applicable. If the screening form indicated that none of the criteria above for definition of complicated pregnancy or delivery was met, the patient was determined to be "uncomplicated" and hence qualified as a 'control' for the purpose of this study. For each near miss case identified, the next two uncomplicated normal vaginal deliveries were selected as controls.

\section{Identification of cause of near-miss}

The primary cause of each case of maternal near-miss was determined by physician review to determine primary and contributing factors associated with maternal near-miss. Primary cause of maternal near-miss was based on the WHO ICD-10 classification,[15] with contributing conditions of anaemia, HIV infection, previous caesarean section, prolonged/ obstructed labor, sickle cell anaemia, and sickle cell crisis. Each case was then reviewed by one of the study investigators to verify cause of near-miss. 
A Qualtrix (Provo, UT) based, structured, interviewer-administered questionnaire was used to record demographic data (including reported material assets, which were combined to calculate a wealth index), pregnancy-related data, and delivery outcome information. Clinical and laboratory data were also abstracted from patients' medical records.

\section{Data analysis}

Data were imported and analyzed with Stata version 13.1 (College Station, Texas). Study data were compared against official hospital tallies to validate the number of deliveries and live births recorded for each month. Maternal near-miss incidence was calculated by dividing the number of maternal near-misses recorded by the number of live births recorded, in keeping with the predominant method in the existing literature[12].

Frequencies and basic descriptive statistics were calculated for all variables, including proportions, means, and standard deviations. Bi-variate statistics and test of associations were performed with Pearson chi square for categorical variables and student's t-test for continuous variables. Assuming an initial alpha of 0.05 , Bonferroni's correction was conducted to identify a level of significance of $\mathrm{p}<0.002$ to account for multiple comparisons. A backward stepwise multiple logistic regression was performed with covariates that were significant in the bivariate analysis to determine the odds ratio (OR) and $95 \%$ confidence interval (CI) for maternal near-miss. The resulting model was re-run using a generalized linear mixed model with site treated as a random effect to account for differences across sites. Coefficients were exponentiated to allow for reporting of odds ratios.

\section{Results}

During the study period, a total of 8,433 live births were recorded across the three study centers, while a total of 288 women were identified to be maternal near-miss (Table 1), yielding an overall maternal near-miss incidence of $3.42 \%$, or an incidence ratio of 34.2 maternal near misses per 1000 live births (95\% CI: 30.2 - 38.1). (Table 2a) There were 79 (27.5\%), 120 (41.7\%) and 89 (30.8\%) near-miss cases at study sites I, II and III respectively. During the same period, a total of 62 maternal deaths were recorded, yielding a maternal mortality incidence ratio of 7.35 (95\% CI 5.5 - 9.2) per 1000 live births. This translates to a near-miss to maternal mortality ratio of 4.6:1(95\% CI: 3.4-6.0).

Table $2 b$ illustrates the primary and underlying causes of maternal near-misses overall, as well as by site. The primary cause of maternal near-miss was severe preeclampsia / eclampsia ( $\mathrm{N}=110,38.2 \%)$, severe haemorrhage $(\mathrm{N}=35,12.1 \%)$, ruptured uterus $(\mathrm{N}=12$, $4.2 \%)$, and maternal sepsis $(\mathrm{N}=10,3.6 \%)$. The secondary or contributing causes of maternal near-miss were; anaemia $(\mathrm{n}=81,28.1 \%)$, hypertensive disorder $(\mathrm{n}=78,27.1 \%)$, infection during pregnancy $(n=49,17.0 \%)$, obstructed labour $(n=39,13.5 \%)$ or other obstetric complications $(\mathrm{n}=41,14.2 \%)$. The distribution of these causes were not consistent: Site 1 contributed a higher proportion of anaemia and pregnancy-related infections than the other two sites; Site 2 had much lower percentages of patients with pre-eclampsia and eclampsia; and Site 3 contributed a disproportionate number of patients with pre-eclampsia and eclampsia as well as hypertensive disorders. (See Table 2b.) 454 women were recruited as healthy controls. The distribution of controls were 149 (32.8\%) from site I, 172 (37.9\%) 
from site II and 133 (29.3\%) from site III respectively. Table 3 illustrates the sociodemographic and health related variables for both cases and controls. There were no significant differences between cases and controls in terms of any of the socio-demographic variables measured, including age, maternal and partner's education, marital status, wealth, or health insurance status. In terms of health-related variables, maternal near-miss was associated with previous caesarean section, non-spontaneous onset of labour for the index birth, multiple births, lower infant birth weight, and fever less than seven days prior to delivery. Mothers who experienced a near-miss were less likely to have delivered a life infant, and they were more likely to have been referred from other facilities to the tertiary centers for care during labour and/or delivery.

Table 4 illustrates the results of the generalized linear mixed model with site as a random effect. Previous caesarean section, multiple pregnancy, and whether the baby was alive at birth were not significant in multivariate analyses and were removed one-by-one to yield the final model shown in Table 4. This model shows that women who experienced a fever within the seven days before birth were nearly six times more likely to have a maternal near-miss than women who did not have a fever in the days leading up to birth (OR $5.9495 \%$ CI 3.65 $-9.68, \mathrm{p}<0.001)$. Women who had been referred from another hospital were 1.5 times more likely to experience a near-miss than women who had not been referred (OR $1.595 \%$ CI $0.99-2.34, \mathrm{p}<0.054)$, however this finding was not statistically significant. Women whose labour began spontaneously were significantly less likely to experience a maternal near-miss than those women whose labour did not start spontaneously. Women with a maternal near-miss were more likely to have a baby with a lower birthweight than controls.

\section{Discussion}

\section{Main findings}

This study assessed cases of maternal near-miss across three tertiary care centers in southern Ghana, finding an overall incidence of 34.2 per 1000 live births. The overall maternal nearmiss-mortality ratio was $4.6: 1$ with slight variability across sites. We found that, overall, severe preeclampsia/eclampsia, haemorrhage, uterine rupture, and sepsis were the leading causes of maternal near-miss, although this varied by site. This is in line with the leading causes of maternal mortality in Ghana which include abortion (19.6\%), postpartum haemorrhage (19.2\%), hypertensive disorders (9.1\%), obstructed labor (7.3\%) and sepsis (6.4\%). [16] The single strongest factor associated with a maternal near-miss was fever within the seven days leading up to birth. Women who reported experiencing a fever in the days before delivery were nearly six times more likely to experience a near-miss than women who did not have a fever, even after controlling for site differences.

\section{Implications for practice}

This study suggests that there is a need to screen for, and pay additional attention to, women who report a fever in the seven days leading up to delivery to avert their progression to potentially life-threatening complications. In view of the known relationship between maternal near-miss and mortality it may be helpful to investigate how febrile morbidity 
contributes to maternal near-miss, especially since the two identified leading causes of maternal near-miss were preeclampsia/eclampsia and haemorrhage.

\section{Strengths and limitations of the study}

This study has several strengths. First, data were collected at three tertiary care centers in Ghana, ensuring diversity in our sample. We also collected data prospectively, not relying upon retrospective chart reviews. We included socio-demographic factors not typically included in clinical studies. However, despite its strengths, our data collection window was limited and thus we cannot explore seasonal or year-to-year differences. We also did not collect quality of care indicators that may have helped contextualize differences found across sites in terms of variability in the number and causes of maternal near-misses.

\section{Interpretation}

This study adds to the literature in several ways. In Ghana, most previous studies have focused on mortality with few studies evaluating morbidity[3-7]. We found the incidence of maternal near-misses across three tertiary care centers dispersed over a wide geographic area to be higher than that previously reported from one hospital in Accra, which identified 94 maternal near-misses out of 3206 live births, or a maternal near-miss incidence of 29.3 per 1000 live births[7]. The maternal near-miss to mortality ratio in our study (4.6:1) is almost twice the 2.5:1 reported despite using the same maternal near-miss criteria in both studies. This observed difference may be due to the inclusion of two other tertiary care centers in Ghana that are outside the capital city. This ratio is also closer to the 5.6:1 reported by some authors from India[16]. Cause of death data were not complete for the maternal deaths that occurred in each of the participating healthcare facilities, thus comparison of cause of death and cause of near-miss within each facility or across the study sites was not possible. However, we obtained national-level cause of maternal death data from the Global Burden of Disease Study[17], allowing us to compare the leading causes of maternal death in Ghana with the leading primary causes of maternal near-miss. We found the leading causes of maternal near-misses at three of the four largest hospitals in the country to be fairly consistent with the leading causes of maternal mortality in Ghana, suggesting that review of cases of maternal near-miss are indeed a viable alternative to review of maternal mortality cases. With decreasing maternal deaths in many places around the world, maternal near-miss identification and maternal near-miss audit is becoming a more useful method to review the quality of care provided.

One interesting aspect of our findings includes the lack of significant association between socio-demographic factors and maternal near-miss. Maternal age, maternal education, husband's education, household size, wealth, parity, gravidity, religion, and marital status were not associated with maternal near-misses. This is different from other studies which found age[18], gravidity[19], maternal education[19], and partner's education[20] to be significantly associated with maternal near-misses. Notably, one study in Brazil found that social and demographic characteristics of the mother were not directly linked to maternal near-miss status, although such factors were linked to differences in care seeking, which was then in turn linked to near-miss status[21]. In our study, care seeking - e.g., number of antenatal visits - was not significantly different between cases of near-miss and controls. 
One possible explanation of the limited role of social factors in our study is that we compared healthy controls against near-misses, rather than comparing near-misses against those women who died. It is possible that women who experience a near-miss are much more similar to healthy controls in terms of socio-demographic characteristics than they are to women who don't survive a life-threatening complication.

\section{Conclusion}

In conclusion this study identified nearly 300 cases of maternal near-miss, reflecting a maternal near-miss incidence of approximately 34 per 1000 live births with approximately five cases of maternal near-miss for every maternal death. Near misses in our study were caused predominantly by hypertensive disorders, haemorrhage, uterine rupture, and sepsis, with the single largest correlate in a multivariate model being the presence of fever in the seven days before delivery. Spontaneous onset of labour was also associated with a lower risk of maternal near miss.

\section{Supplementary Material}

Refer to Web version on PubMed Central for supplementary material.

\section{Acknowledgement}

We wish to acknowledge the authorities of the KBTH, KATH and CCTH for their immense support during the study. We also wish to acknowledge Miss Elorm Kudzawu, Zelda Arku, Magdalene Torto and Dominic Nyarko for their roles in participants recruitment and administrative support in conducting this study

Funding

The study was funded by the U.S. National Institutes of Health, 1-D43-TW-009353-01 grant provided to the University of Michigan. The funding agency played no role in study design, data analysis, writing the manuscript or the decision to publish this manuscript

\section{References}

1. World Health Organization. Trends in maternal mortality 1990-2013. Available at http:// apps.who.int/iris/bitstream/10665/112682/2/9789241507226_eng.pdf?ua=1 2014 Accessed September 29, 2017.

2. WHO, UNICEF, UNFPA, The World Bank. Trends in Maternal Mortality: 1990-2008. http:// whqlibdoc.who.int/publications/2010/9789241500265_eng.pdf. Published 2010.

3. Zakaria AY, Alexander S, van Roosmalen J, Buekens P, Kwawukume EY, Frimpong P. Reproductive age mortality survey (RAMOS) in Accra, Ghana. Reprod Health 2009; 6-7 [PubMed: 19386134]

4. Adu-Bonsaffoh K, Oppong SA, Bilinla G, Obed SA. Maternal deaths attributable to hypertensive disorders in a tertiary hospital in Ghana. Int J Obstet Gynecol 2013, 123(2): 110-3

5. Gumanga SK, Kolbila DZ, Gandau BBN, Munkaila A, Malechi H. Trends in maternal mortality in Tamale Teaching hospital. Ghana Med J 2011, 45(3): 105-10 [PubMed: 22282577]

6. Asamoah BO, Moussa KM, Stafstrom M, Musinguzi G. Distribution of causes of maternal mortality among different socio-demographic groups in Ghana; a descriptive study. BMC Public Health 2011 1; 11(1): 159 [PubMed: 21392387]

7. Tuncalp O, Hindin MJ, Adu-Bonsaffoh K, Adanu RM. Assessment of maternal near-miss and quality of care in a hospital-based study in Accra, Ghana. Int J Gynecol Obstet 2013, 123; 58-63 
8. Tuncalp O, Hindin MJ, Adu-Bonsaffoh K, Adanu RM. Listening to women's voices: the quality of care of women experiencing severe maternal morbidity in Accra, Ghana. PLoSONE 7(8): e44536.doi:10.1371/journal.pone.0044536

9. WHO. Beyond the numbers: Reviewing maternal deaths and complications to make pregnancy safer http://www.who.int/maternal_child_adolescent/documents/9241591838/en/

10. Tuncalp O, Hindin M, Souza P, Cho D, Say L. The prevalence of maternal near-miss: a systematic review. BJOG 2012; 119: 653-661 [PubMed: 22489760]

11. Say L, Souza JP, Pattinson RC. Maternal near-miss: towards a standard tool for monitoring quality of maternal health care. Best Practice \& Research Clinical Obstet and Gynecol 2009, 23: 287-296

12. Kaye DK, Kakaire O, Osinde MO. Systematic review of the magnitude and case fatality ratio for severe maternal morbidity in Sub-Saharan Africa between 1995 - 2010. BMC Pregnancy Childbirth. 20119 28;11:65. doi: 10.1186/1471-2393-11-65. [PubMed: 21955698]

13. Ghana Statistical Service. 2010 Population and Housing Census.

14. Souza JP, Cecatti JG, Haddad SM, Parpinelli MA, Costa ML, Katz L, et al. Brazilian Network for Surveillance of Severe Maternal Morbidity Group; The WHO maternal near-miss approach and the maternal severity index model (MSI): tools for assessing the management of severe maternal morbidity. PLoS One. 2012;7(8):e44129. doi: 10.1371/journal.pone.0044129. [PubMed: 22952897]

15. World Health Organization, editor. The WHO application of ICD-10 to deaths during pregnancy, childbirth and the puerperium, IDC MM. Geneva: World Health Organization; 201268 p.

16. Kassebaum NJ, Bertozzi-Villa A, Coggeshall MS, Shakelford KA, Steiner C, Heuton KR, et al. Global, regional, and national levels and causes of maternal mortality during 1990-2013: a systematic analysis for the Global Burden of Disease Study 2013. Lancet. 2014;384(9947):9801004. doi:10.1016/S0140-6736(14)60696-6. [PubMed: 24797575]

17. Roopa PS, Verma S, Rai L, Kumar P, Pai MV, Shetty J. "Near Miss" Obstetric Events and Maternal Deaths in a Tertiary Care Hospital: An Audit. Journal of Pregnancy. 2013;

18. Pafs J, Musafili A, Binder-Finnema P, Klingberg-Allvin M, Rulisa S, Essen B. Beyond the numbers of maternal near-miss in Rwanda - a qualitative study on women's perspectives on access and experiences of care in early and late stage pregnancy. BMC Pregnancy \& Childbirth. 2016; 16: 257. [PubMed: 27590589]

19. Nakumuli A, Nakubulwa S, Kakaire O, Osinde MO, Mbalinda SN, Nabirye RC, et al. Maternal near misses from two referral hospitals in Uganda: a prospective cohort study on incidence, determinants and prognostic factors. BMC Pregnancy \& Childbirth. 2016; 16: 24. [PubMed: 26821716]

20. Nansubuga E, Ayiga N, Moyer CA. Prevalence of maternal near miss and community-based risk factors in Central Uganda. Int J Gynecol Obstet. 2016; 135: 214-220.

21. Domingues RMSM, Dias MAB, Schilithz AOC, Leal MDC. Factors associated with maternal near miss in childbirth and the postpartum period: findings from the birth in Brazil National Survey, 2011-2012. Reproductive Health. 2016; 13 (Suppl 3): 115. [PubMed: 27766973] 
Table 1:

Identification of maternal near-miss in three tertiary hospitals in southern Ghana

\begin{tabular}{|c|c|}
\hline $\begin{array}{l}\text { Symptom-based criteria } \\
\text { (yes to any of the following: severe haemorrhage }(n=19) \text {, severe preeclampsia }(n=30) \text {, eclampsia }(n=84) \text {, sepsis or systemic } \\
\text { infection }(n=12) \text {, ruptured uterus }(n=1))\end{array}$ & $146(19.7)$ \\
\hline $\begin{array}{l}\text { Intervention-based criteria } \\
\text { (yes to any of the following: use of blood products }(n=98) \text {, laparotomy }(n=67) \text {, admission to ICU }(n=102) \text { ) }\end{array}$ & $189(25.5)$ \\
\hline $\begin{array}{l}\text { Organ-dysfunction-based criteria } \\
\text { (yes to specific indicators for cardiovascular }(n=28) \text {, respiratory }(n=23) \text {, renal }(n=5) \text {, haematologic }(n=16) \text {, hepatic }(n=4) \text {, } \\
\text { neurologic }(n=6) \text {, and uterine dysfunction }(n=5))\end{array}$ & $60(8.1)$ \\
\hline Met no criteria & $454(61.2)$ \\
\hline Met one category of criteria & $202(27.22)$ \\
\hline Met two categories of criteria & $64(8.6)$ \\
\hline Met three categories of criteria & $22(3.0)$ \\
\hline
\end{tabular}


Table 2a:

Maternal near-miss incidence and incidence ratio at three tertiary hospitals.

\begin{tabular}{|l|l|l|l|l|}
\hline \multirow{2}{*}{} & \multicolumn{3}{|c|}{ Study sites } & Total \\
\cline { 2 - 5 } & I & II & III & \\
\hline Number of live births & 1149 & 3596 & 3688 & 8433 \\
\hline Number of maternal deaths & 11 & 32 & 19 & 62 \\
\hline Number of maternal near-miss cases identified & 79 & 120 & 89 & 288 \\
\hline Maternal near-miss incidence (per 1000 live births) (95\% CI) & $68.7(53.6,83.9)$ & $33.4(27.4,39.3)$ & $24.1(19.1,29.1)$ & $34.2(30.2,38.1)$ \\
\hline Maternal mortality per 1000 live births (95\% CI) & $9.5(3.9,15.2)$ & $8.9(5.8,11.9)$ & $5.5(2.8,7.4)$ & $7.4(5.5,9.2)$ \\
\hline Maternal near-miss : mortality ratio (95\% CI) & $7.2: 1(3.8,13.6)$ & $3.8: 1(2.5,5.5)$ & $4.4: 1(2.8,7.7)$ & $4.6: 1(3.4,6.0)$ \\
\hline
\end{tabular}


Table $\mathbf{2 b}$ :

Primary and contributing causes of maternal near-misses overall and by study site

\begin{tabular}{|l|l|l|l|l|l|}
\hline \multirow{2}{*}{} & \multicolumn{4}{|c|}{ Study sites } & Overall \\
\cline { 2 - 5 } & I (N=79) N (\%) & II (N=120) N (\%) & III (N=89) N (\%) & $(\mathbf{N = 2 8 8 ) ~ N ~ ( \% ) ~}$ \\
\hline Primary Causes & $38(48.1)$ & $9(7.5)$ & $63(70.8)$ & $110(38.2)$ \\
\hline Pre-eclampsia and Eclampsia & $3(10.1)$ & $22(18.3)$ & $5(5.6)$ & $35(12.1)$ \\
\hline Hemmorhage & $5(6.3)$ & $5(4.2)$ & $2(2.3)$ & $12(4.2)$ \\
\hline Ruptured Uterus & $6(7.6)$ & $3(2.5)$ & $1(1.2)$ & $10(3.8)$ \\
\hline Sepsis & $45(56.9)$ & $15(12.5)$ & $21(23.6)$ & $81(28.1)$ \\
\hline Underlying or contributing causes & $8(6.7)$ & $61(68.5)$ & $78(27.1)$ \\
\hline Anemia & $9(11.4)$ & $1(0.8)$ & $3(3.4)$ & $49(17.0)$ \\
\hline Hypertensive disorders & $45(57.0)$ & $19(15.8)$ & $7(7.9)$ & $39(13.5)$ \\
\hline Infection during pregnancy & $13(16.5)$ & $4(3.3)$ & $35(39.3)$ & $41(14.2)$ \\
\hline Obstructed labor & $2(2.5)$ & & \\
\hline Other obstetric complications & &
\end{tabular}


Table 3:

Socio-demographic and health-related variables for maternal near-miss cases and controls at three tertiary hospitals in southern Ghana

\begin{tabular}{|l|l|l|l|}
\hline Variable name $($ Total N=742) & Cases $(\mathbf{N = 2 8 8 )}$ Mean $(\mathbf{S D})$ & Controls (N=454) Mean $(\mathbf{S D})$ & T -test statistic $(\mathbf{p}$-value) \\
\hline Maternal age $(\mathrm{N}=445)$ & $29.7(6.4)$ & $28.8(6.3)$ & $-1.47(0.15)$ \\
\hline Maternal education (years) $(\mathrm{N}=447)$ & $8.9(4.0)$ & $8.9(4.4)$ & $-0.11(0.91)$ \\
\hline Husband's education (years) $(\mathrm{N}=351)$ & $10.5(3.9)$ & $10.5(3.8)$ & $0.21(0.83)$ \\
\hline Assets (on 0-16 point scale) $)^{*}(\mathrm{~N}=327)$ & $9.1(3.4)$ & $9.2(3.4)$ & $0.31(0.75)$ \\
\hline Mean number of prior births $(\mathrm{N}=732)$ & $1.7(1.7)$ & $1.7(1.7)$ & $0.11(0.91)$ \\
\hline Number of ANC visits $(\mathrm{N}=351)$ & $6.7(2.4)$ & $6.9(2.6)$ & $0.83(0.40)$ \\
\hline Infant birthweight $(\mathrm{kg})(\mathrm{N}=678)$ & $2.653(0.803)$ & $3.080(0.597$ & $7.91(<\mathbf{0 . 0 0 1})$ \\
\hline
\end{tabular}

\begin{tabular}{|l|l|l|l|}
\hline & Cases \% $(\mathbf{N})$ & Controls \% $(\mathbf{N})$ & Chi-Square statistic (P value) \\
\hline Married (N=742) & $38.5(111)$ & $43.4(197)$ & $1.71(0.19)$ \\
\hline Polygamous marriage (N=742) & $3.5(10)$ & $1.8(8)$ & $2.17(0.14)$ \\
\hline Has national health insurance (N=447) & $93.6(147)$ & $96.2(279)$ & $1.51(0.22)$ \\
\hline Delivered in a healthcare facility (N=444) & $99.4(154)$ & $98.9(286)$ & $0.17(0.67)$ \\
\hline Previous c-section (N=740) & $28.8(83)$ & $13.7(62)$ & $25.46, \mathbf{p}<\mathbf{0 . 0 0 1}$ \\
\hline Mode of delivery index pregnancy (N=742) & & & $3.4, \mathrm{p}=0.18$ \\
\hline Vaginal delivery & $86.5(249)$ & $89.7(407)$ & \\
\hline C-section & $8.7(25)$ & $7.9(36)$ & \\
\hline Spontaneous labour (N=712) & $47.4(129)$ & $89.09(392)$ & $149.4, \mathbf{p}<\mathbf{0 . 0 0 1}$ \\
\hline Fever <7 days before labour (N=705) & $39.1(104)$ & $13.4(59)$ & $61.3, \mathbf{p}<\mathbf{0 . 0 0 1}$ \\
\hline Anaemia during pregnancy (N=702) & $35.9(95)$ & $33.9(148)$ & $0.04, \mathrm{p}=0.85$ \\
\hline Multiple pregnancy (N=726) & $7.1(20)$ & $3.8(17)$ & $3.72, \mathrm{p}=0.05$ \\
\hline Live infant at birth (N=715) & $91.1(255)$ & $97.2(423)$ & $13.2, \mathbf{p}<\mathbf{0 . 0 0 1}$ \\
\hline Referred from other healthcare facility (N=731) & $49.1(139)$ & $35.9(161)$ & $12.4, \mathbf{p}<\mathbf{0 . 0 0 1}$ \\
\hline
\end{tabular}

Assets include aggregate list of 16 different material goods owned or available within the household, including car/truck, motorcycle, bicycle, electricity, solar light, refrigerator, television, DVD/VCR, radio, sewing machine, stereo system, electric/box iron, fan, mobile phone, electric/gas cookstove, donkey cart/push truck, kerosene stove, personal computer 
Table 4:

Generalized linear mixed model (with site as a random effect) for predictors of maternal near-miss at three tertiary hospitals in southern Ghana

\begin{tabular}{|l|l|l|l|l|l|}
\hline Variable & Odds Ratio & Standard error & $\mathbf{Z}$ & P value & 95\% CI \\
\hline Infant birthweight & 0.99 & 0.0001 & -5.07 & $<\mathbf{0 . 0 0 1}$ & $0.998-0.999$ \\
\hline Spontaneous onset of labor & 0.09 & 0.245 & -9.81 & $<\mathbf{0 . 0 0 1}$ & $0.056-0.146$ \\
\hline Fever in 7 days before delivery & 5.94 & 0.248 & 7.16 & $<\mathbf{0 . 0 0 1}$ & $3.649-9.681$ \\
\hline Referred from another facility & 1.52 & 0.530 & 1.93 & 0.054 & $0.992-2.344$ \\
\hline
\end{tabular}

